\title{
Avaliação da Toxicidade do extrato bruto metanólico de Baccharis dracunculifolia por meio do bioensaio com Artemia salina
}

\section{Palloma de Almeida Soares Hocayen ${ }^{15}$, Letícia Antonelo Campos ${ }^{2}$, Márcia Thaís Pochapski $^{3}$ \& Carlos Ricardo Maneck Malfatti ${ }^{4}$}

Enviado em junho de 2012; aceito em novembro de 2012.

\section{Resumo}

As plantas medicinais vêm sendo mundialmente empregadas na medicina popular. Dentro do gênero Baccharis, a espécie Baccharis dracunculifolia se destaca, sendo utilizada para vários males. $\mathrm{O}$ uso indiscriminado de plantas sem qualquer conhecimento fitoquímico, farmacológico e principalmente toxicológico é de grande preocupação para a saúde. Assim, encontrar uma concentração segura e não letal para o uso terapêutico constitui-se uma etapa de grande importância. Desse modo, buscouse neste estudo avaliar a toxicidade do extrato bruto metanólico de Baccharis dracunculifolia frente às larvas de Artemia salina. Os resultados mostraram uma concentração letal média $\left(\mathrm{CL}_{50}\right)$ de 1008,51ug/mL e 921,32ug/mL em 24 e $48 \mathrm{~h}$ respectivamente, sendo necessária uma concentração muito alta para ser considerada letal. Palavras-chave: Toxicidade, Baccharis dracunculifolia, Artemia salina.

\section{Abstract}

(Evaluation of the toxicity of the methanolic crude extract of Baccharis dracunculifolia on Artemia salina). Medicinal plants have been used in folk medicine worldwide. Within the genus Baccharis, species Baccharis dracunculifolia stands out, being used for several diseases. The indiscriminate use of plants without any knowledge phytochemical, pharmacological and toxicological mainly is of great concern to health. Finding a safe and non-lethal concentration to the therapeutic use constitutes an important step. Thus, this study sought to evaluate the toxicity of the methanolic crude extract of Baccharis dracunculifolia front of the larvae of Artemia

DOI: http://dx.doi.org/10.5007/2178-4574.2012n41p23

${ }^{1}$ Mestre em Ciências Biológicas, Universidade Estadual de Ponta Grossa, Paraná, Ponta Grossa, Brasil.

${ }^{2}$ Mestranda em Odontologia, Universidade Estadual de Ponta grossa, Paraná, Ponta Grossa, Brasil.

${ }^{3}$ Departamento de Odontologia, Universidade Estadual de Ponta Grossa, Paraná, Ponta Grossa, Brasil.

${ }^{4}$ Departamento de Educação Física, Universidade Estadual do Centro-Oeste, Paraná, Irati, Brasil.

${ }^{5}$ Autor para correspondência: pallomaas@yahoo.com.br

\section{$(\mathrm{cc}) \mathrm{BY}-\mathrm{Ne}$}

Este artigo é de Acesso Livre, disponibilizado sob os termos da

Creative Commons Attribution 3.0 Unported License (http://creativecommons.org/licenses/by/3.0/) que permite uso não-comercial, distribuição e reprodução em qualquer meio, desde que este trabalho original seja devidamente citado. 
salina. The results showed a median lethal concentration (LC 50) of $1008,51 \mathrm{ug} / \mathrm{mL}$ e $921,32 \mathrm{ug} / \mathrm{mL}$ after $24 \mathrm{~h}$ and $48 \mathrm{~h}$ respectively, being necessary a very high concentration be considered lethal.

Key words: Toxicity, Baccharis dracunculifolia, Artemia salina.

\section{Introdução}

A utilização das plantas medicinais, para tratamentos de doenças, é uma prática muito antiga, sendo encontrada ao longo da história em todas as populações mundiais e em diferentes grupos étnicos conhecidos. A partir deste uso popular foram encontrados diversos medicamentos utilizados na medicina tradicional, constituindose um importante recurso para a descoberta de novos fármacos (Martins 2000, Bussmann 2009).

No Brasil a população tem um importante conhecimento etnobotânico e etnofarmacológico em decorrência da miscigenação do povo, decorrente dos colonizadores e escravos vindos do oriente somados ao conhecimento indígena local (Ferreira 2002). Tais observações populares em relação ao uso e a eficácia de plantas medicinais contribuem de forma relevante para a divulgação das propriedades terapêuticas dos vegetais, principalmente em virtude dos efeitos medicinais que produzem (Maciel et al. 2002).

O gênero Baccharis foi descrito por Linné em 1753, composto por mais de 400 espécies, com grande dispersão pela América Latina, principalmente no Brasil, destacando os estados do Paraná, Santa Catarina, São Paulo e Rio Grande do Sul (Bremer 1994). Cerca de $98 \%$ dos gêneros são constituídos por plantas de pequeno porte, sendo encontradas em todos os tipos de habitat, mas principalmente nas regiões tropicais montanhosas (Joly 1998).

No Brasil estão descritas pelo menos 120 espécies de Baccharis. Parte considerável das espécies já identificadas foi utilizada em estudos químicos relevantes, sendo que algumas, em virtude dos resultados apresentados, foram aplicadas em estudos com ensaios biológicos (Barroso et al. 2002, Verdi et al. 2005).

$\mathrm{O}$ gênero é tido como fonte de vários compostos, tais como: sesquiterpenos, diterpenos, triterpenos e flavonóides (Jarvis et al. 1991). Os flavonóides juntamente com os diterpenos são os constituintes mais encontrados no gênero Baccharis (Emerenciano et al. 2001), sendo utilizados para vários males como fonte terapêutica (Jakupovic et al. 1990).

Dentre as espécies do gênero destaca-se a Baccharis dracunculifolia pertence à família Asteraceae que é o grupo sistemático mais numeroso dentro das Angiospermas, compreendendo cerca de 1.100 gêneros e 25.000 espécies, distribuídas em regiões tropicais, subtropicais e temperadas (Bremer 1994, Barroso et al. 2002). No Brasil a família está representada por cerca de 180 gêneros e 1.900 espécies (Barroso et al. 2002). 
A espécie tem grande uso popular, os seus ramos e folhas são utilizados para combater vários males, dentre eles: distúrbios gástricos, cansaço físico, inapetência, afecções febris e debilidade orgânica, além de ser utilizada em feridas e processos inflamatórios (Menezes 2005).

A planta medicinal Baccharis dracunculifolia tem sido a fonte botânica mais importante para produção de própolis verde, além de seus diferentes extratos (Kumazawa et al. 2003, Park et al. 2004, Midorikawa et al. 2001) para os quais têm sido atribuídas inúmeras atividades biológicas, tais como: antimicrobiana, antioxidante, anti-inflamatória (Fabri et al. 2011, Menezes 2005).

Estudos com plantas medicinais buscam identificar substâncias ativas, ressaltando que o uso indiscriminado das plantas pela população pode trazer riscos, por isso é importante a realização de testes de toxicidade na busca de uma terapêutica eficaz, trazendo assim um maior conforto e melhor qualidade de vida aos pacientes.

O teste de toxicidade contra a Artemia salina é um ensaio biológico considerado como uma das ferramentas mais utilizadas para a avaliação preliminar de toxicidade. Desta forma, A. salina tem sido utilizada como um organismo alvo para detectar compostos bioativos em extratos de plantas (Alves et al. 2000), a toxicidade para este crustáceo tem demonstrado uma boa correlação com a atividade citotóxica contra tumores humanos (Mclaughlin et al. 1991) e atividade contra o Trypanossoma cruzi (Alves et al. 2000), protozoário causador da doença de Chagas.

Sendo assim, objetivou-se com este estudo avaliar a toxicidade aguda do extrato metanólico de Baccharis dracunculifolia frente a larvas de Artemia salina, visando a segurança e eficácia de sua utilização em ensaios pré-clínicos e clínicos.

\section{Material e Métodos}

Foram coletados aproximadamente $5 \mathrm{~kg}$ de planta fresca, no Campus da Universidade Estadual de Ponta Grossa, em agosto de 2010. A exsicata da planta foi depositada no Herbário da Universidade Estadual de Ponta Grossa - HUPG e registrada com o número 18173.

As folhas e galhos foram secos ao ar durante dez dias, moídos e submetidos à extração por maceração em metanol. O líquido de maceração foi, posteriormente, filtrado. O filtrado restante foi rotaevaporado e em seguida liofilizado. $\mathrm{O}$ extrato obtido será utilizado posteriormente em ensaio pré-clínico.

A toxicidade do extrato foi testada no modelo da A. salina baseada na técnica descrita por Meyer et al. (1982). Foram utilizadas 60mg do extrato bruto dissolvidos em $30 \mathrm{~mL}$ de metanol. As soluções foram homogeneizadas e para o volume final foram adicionados $5 \mathrm{~mL}$ de solução salina de sal marinho, obtendo-se concentrações de 25, 50,100,150,250, 500, 750 e $1000 \mu \mathrm{g} / \mathrm{ml}$.

As larvas de A. salina foram incubadas, nas diversas concentrações do extrato bruto dissolvido, durante $24 \mathrm{~h}$ sob iluminação artificial e temperatura controlada para eclodirem. Posteriormente foram transferidas 10 larvas para tubos de ensaio contendo solução salina (controle negativo), dicromato de potássio $0,1 \%$ 
(controle positivo) e o extrato nas diferentes concentrações. Após 24 e $48 \mathrm{~h}$ contaramse os microcrustáceos mortos. $\mathrm{A} \mathrm{CL}_{50}$ foi obtida por meio do gráfico da percentagem de animais mortos em função do logarítimo da dose testada. O teste foi realizado em triplicata.

Para obtenção dos valores de $\mathrm{CL}_{50}$ foi utilizada a análise PROBIT por meio do software STATPLUS® 2007 (Melo 2008).

\section{Resultados e Discussão}

Os resultados demonstraram que para 24 e 48 horas $(1008,51 \mathrm{ug} / \mathrm{mL}$ e 921,32ug/mL respectivamente) de incubação (Figuras 1 e 2), o extrato de Baccharis dracunculifolia precisou de uma alta concentração para ser considerado letal. Por outro lado, o controle positivo (dicromato de potássio), que possui a capacidade de matar as larvas de Artemia salina, obteve alta toxicidade, matando um número elevado de larvas de A. salina no período de $24 \mathrm{~h}$, concentração de $69,33 \mathrm{ug} / \mathrm{mL}$ (Figura 3). No período $48 \mathrm{~h}$ a estimativa da concentração letal do dicromato de potássio foi negativa $(-555,02 \mathrm{ug} / \mathrm{mL})$, (Figura 4) neste caso entende-se que uma mínima concentração seria suficiente para matar $50 \%$ dos organismos. A comparação realizada entre o extrato de $B$. dracuculifolia e dicromato de potássio em relação aos sobreviventes (Figuras 5 e 6), reafirma o que já foi exposto acima, que a concentração letal de $B$. dracunculifolia precisa ser alta e a de dicromato baixa para matar grande quantidade de larvas.

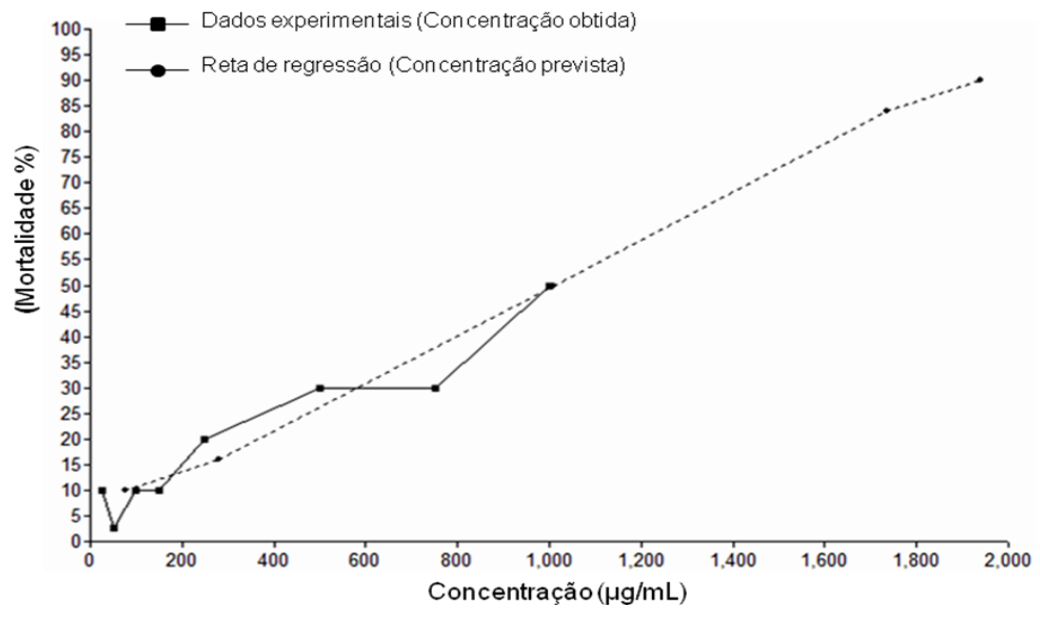

CL50 = 1008,51 $\mu \mathrm{g} / \mathrm{mL}$ (Análise de Regressão Probit). CL - Concentração letal.

Figura 1. Curva concentração-resposta da mortalidade de Artemia salina ao extrato metanólico de Baccharis dracunculifolia após 24 horas. 


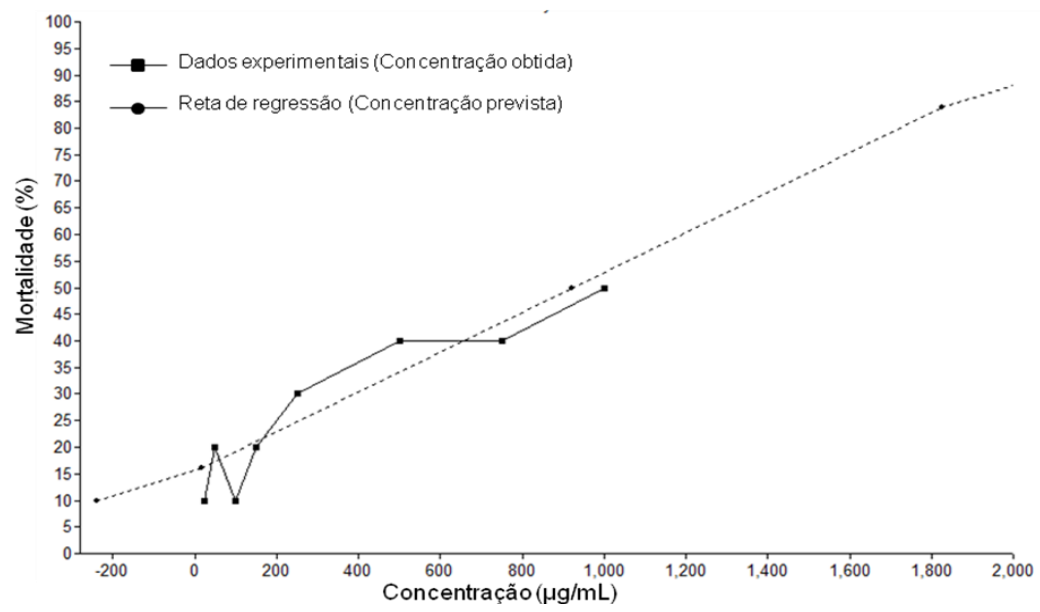

CL50 = 921,32 $\mu \mathrm{g} / \mathrm{mL}$ (Análise de Regressão Probit). CL - Concentração letal.

Figura 2. Curva concentração-resposta da mortalidade de Artemia salina ao extrato metanólico de Baccharis dracunculifolia após 48 horas.

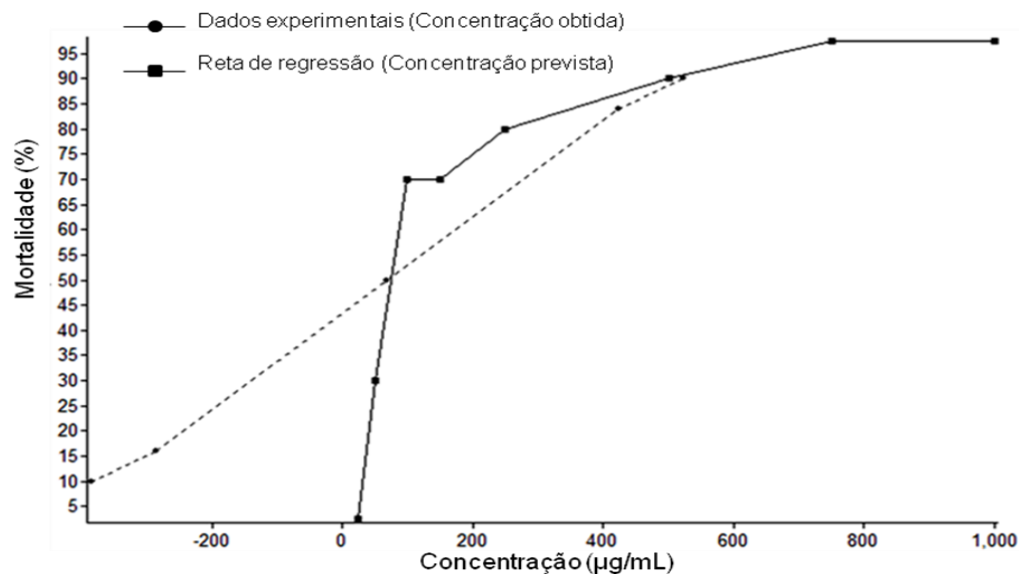

CL50 = 69,33 $\mu \mathrm{g} / \mathrm{mL}$ (Análise de Regressão Probit). CL - Concentração letal.

Figura 3. Curva concentração-resposta da mortalidade de Artemia salina ao dicromato de potássio (controle positivo) após 24 horas. 


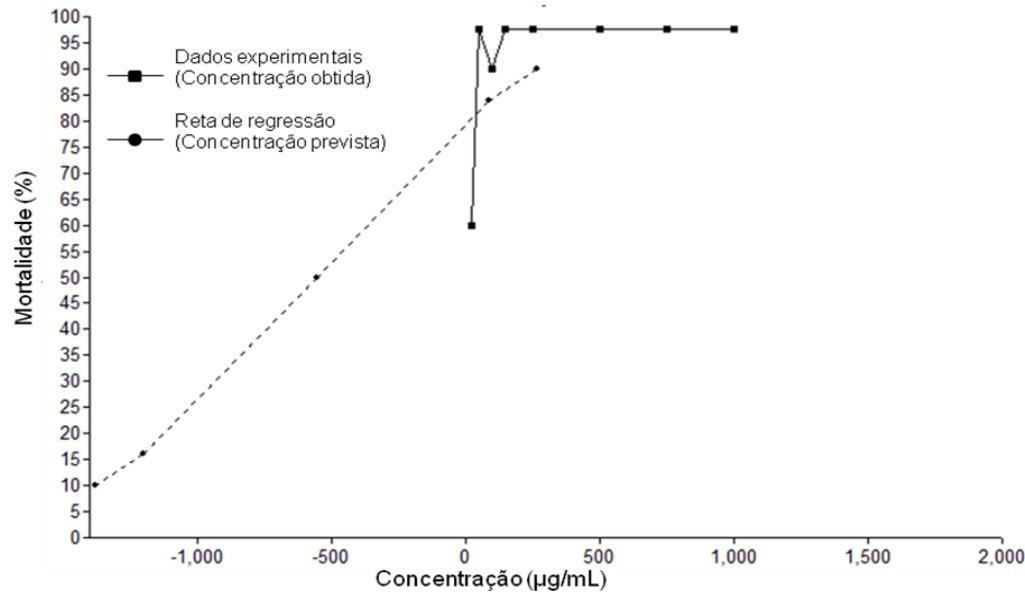

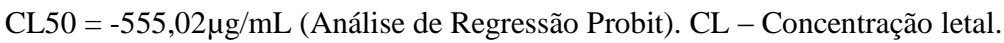

Figura 4. Curva concentração-resposta da mortalidade de Artemia salina ao dicromato de potássio (controle positivo) após 48 horas.

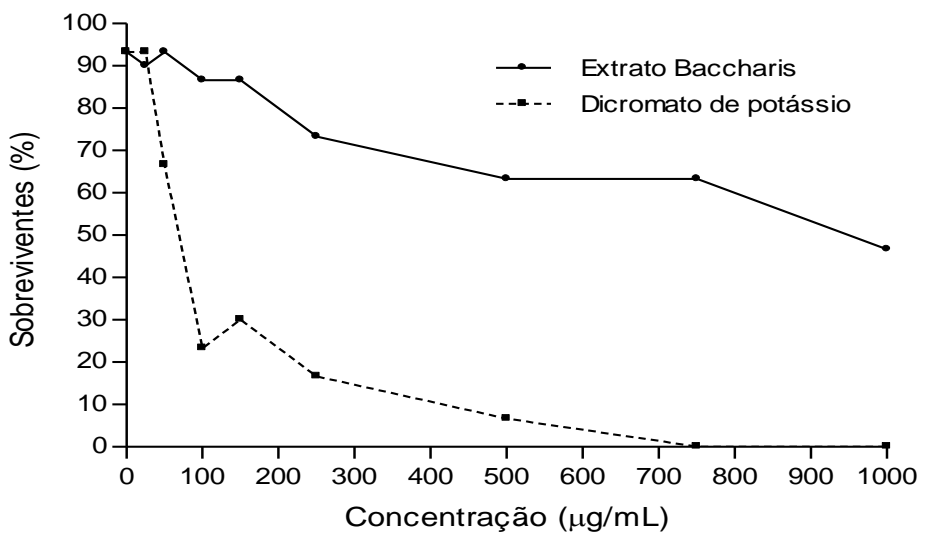

Figura 5. Curvas dose-resposta da sobrevivência de Artemia salina ao extrato metanólico de Baccharis dracunculifolia e dicromato de potássio (controle positivo) após 24 horas. 


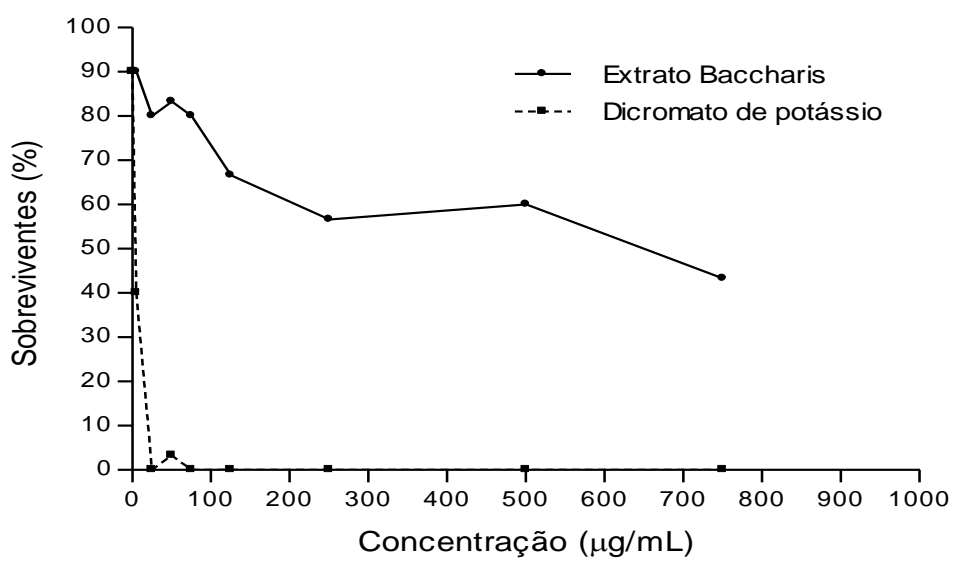

Figura 6. Curvas dose-resposta da sobrevivência de Artemia salina ao extrato metanólico de Baccharis dracunculifolia e dicromato de potássio (controle positivo) após 48 horas.

Esses resultados anuem com outros dados da literatura que ressaltaram que a própolis de $B$. dracunculifolia apresenta uma toxicidade dependente da concentração sobre células NIH-3T3 (linhagem celular estabelecida a partir de culturas de embriões) de fibroblastos de ratos, assim como o extrato, óleo e própolis de B. dracunculifolia não mostraram atividade citotóxica contra células HEp2, linhagem celular de carcinoma laríngeo humano (Funari 2007, Bufalo 2010).

Em diversos estudos realizados com o extrato de $B$. dracunculifolia em ensaios pré-clínicos, foram utilizadas doses variando de 50 a $500 \mathrm{mg} / \mathrm{Kg}$, em ensaios anti-ulcerogênico, anti-inflamatório, antimicrobiano não tendo sido encontrado toxicidade em nenhum destes testes com animais (Fabri et al. 2011, Menezes 2005, Lemos et al. 2007), assim como, não foram encontrados dados na literatura referentes à intoxicação da população com a utilização desta planta.

Os resultados demonstram que o extrato metanólico de $B$. dracunculifolia apresenta baixa toxicidade frente as larvas de Artemia salina, podendo ser utilizado em alta concentração com uma dose segura de $400 \mathrm{mg} / \mathrm{Kg}$, em bioensaios in vitro e in vivo.

\section{Referências}

Alves, T.M.A.; Silva, A.F.; Brandão, M.; Grandi, T.S.M.; Smânia, E.F.A.; Smânia Jr., A.; Zani, C.L. 2000. Biological screening of Brazilian medicinal plants. Memórias do Instituto Oswaldo Cruz 95: 367-373. 
Barroso, G.M.; Peixoto, A.L.; Ichaso, C.L.F.; Costa, C.G.; Guimarães, E.F.; Lima, H.C. 2002. Sistemática de Angiospermas do Brasil. 2 ed. Viçosa: Imprensa Universitária, Universidade Federal de Viçosa. v.1.

Bremer, K. 1994. Asteraceae: Cladistics and classification. Timber Press: Portland. Búfalo, M.C.; Candeias, J.M.G.; Bastos, J.K.; Sforcin, J.M. 2010. In vitro cytotoxic activity of Baccharis dracunculifolia and propolis against HEp-2 cells. Natural Product Research (Print) 24: 1710-18.

Bussmann R.W. 2004. Ethnobothany and Biodiversity and Conservation. http://www.olorien.org/rbussmann/academicCV.php. Acesso em: 05 dez. 2010. Emerenciano,V.P.; Kaplan, M.A.C.; Bonfanti, M.R.M.; Ferreira, Z.S.; Comegno, L.M.A. 2001. Evolution of Sesquiterpene Lactones in Asteraceae. Biochemical Systema Ecs and Ecology 14(6): 585-589.

Fabri, R.L.; Nogueira, M.S.; Dutra, L.B.; Bouzada, M.L.M.; Scio, E. 2011. Potencial antioxidante e antimicrobiano de espécies da família Asteraceae. Rev. Bras. PI. Med.13: 183-189.

Ferreira, S.H. 2002. Academia Brasileira de Ciências: medicamentos a base de plantas medicinais. http:www.abc.org.br/arquivos/medicamentos.pdf. Acesso em: maio 2010.

Funari, C.S.; Ferro, V.O.; Mathor, M.B. 2007. Análise de própolis de Baccharis dracunculifolia DC. (Compositae) e seus efeitos sobre fibroblastos de rato. Journal of Ethnopharmacology 111(2): 206-212.

Jakupovic, J.; Schuster, A.; Ganzer, U.; Bohlmann, F.; Boldt, P.E. 1990. Sesqui- and diterpenes fron Baccharis species. Phytochemistry 29(7):2217-2222.

Jarvis, B.B.; Rejali, N.M.; Schenkel, E.P.; Barros, C.S.; Matzenbacher, N.I. 1991. Trichothecene mycotoxins from Brazilian Baccharis species. Phytochemistry 30(3): 789-797.

Joly, A.B. 1998. Botânica: introdução a taxonomia vegetal. São Paulo: Cia Editora Nacional.

Kumazawa, S.; Yoneda, M.; Shibata, I.; Kanaeda, J.; Hamasaka, T.; Nakayama, T. 2003. Direct evidence for the plant origin of Brazilian propolis by the observation of honeybee behavior and phytochemical analysis. Chemical and Pharmaceutical Bulletin 51(6): 740-742.

Lemos, M.; Barros, M.P.; Sousa, J.P.B.; Da Silva Filho, A.A.; Bastos, J.K.; De Andrade, S.F. 2007. Baccharis dracunculifolia, the main botanical source of Brazilian green própolis, displays antiulcer activity. J. Pharm. Pharmacol. 59 (4): 603-608.

Maciel, M.A.M.; Pinto, A.C.; Viga J.R. 2002. Plantas medicinais: a necessidade de estudos multidisciplinares. Química Nova 25(3): 429-438.

Martins, E.R.; Castro, D.M.; Castellani, D.C.; Dias, J.E. 2000. Plantas medicinais. Viçosa: Editora UFV. 
McLaughlin, J.L.; Chang, C.J.; Smith, D.L. 1991. "Bench-top" bioassays for the discovery of bioactive natural products. Studies in Natural Product Chemistry 9:383-409.

Melo, A.F.M.; Nascimento, J.E.; Lima e Silva, T.C.; Veras Filho, J.; Santos, E.M.; Albuquerque, U.P.; Amorim, E.L.C. 2008. Estudo fitoquímico e bioensaio toxicológico frente a larvas de Artemia salina Leach. de três espécies medicinais do gênero Phyllanthus (Phyllanthaceae), Rev. Ciênc. Farm. Básica Apl. 29(2): 145-150.

Menezes, H. 2005. Avaliação da atividade antiinflamatória do extrato aquoso de Baccharis dracunculifolia (Asteraceae). Arquivos do Instituto Biológico de São Paulo 72: 1-64.

Meyer, B.N.; Ferrigni, N.R.; Putnam, L.B.; Jacobsen, L.B.; Nichols de Mclaughlin, J.L. 1982. Brine shrimp: a convenient general bioassay for active plant constituents. J Med Plant Res. 45: 31- 34.

Midorikawa, K.; Banskota, A.H.; Tezuka, Y.; Nagaoka, T.; Matsushige, K.; Message, D.; Huertas, A.A.; Kadota, S. 2001. Liquid chromatography-mass spectrometry analysis of propolis. Phytochemical Analysis 12(6): 366-373.

Park, Y.K.; Alencar, S.M.; Aguiar, C.L.; Guzmán, J.P. 2004. Chemical constituents in Baccharis dracunculifolia as the main botanical origin of Southeastern Brazilian propolis J. Agric. Food Chem. 52: 1100-1103.

Verdi, L.G.;Brighente, I.M.C.; Pizzolatti, M.G. 2005. Gênero Baccharis (Asteraceae): aspectos químicos, econômicos e biológicos. Quím. Nova 28(1): 85-94. 